monográfico: Trasplante Renal. Experiencia del Hospital Clínico de Barcelona

Arch. Esp. Urol., 58, 6 (547-551), 2005

\title{
ENFERMEDADES GLOMERULARES Y TRASPLANTE RENAL DE DONANTE VIVO.
}

Rita Guerra ${ }^{1}$, Alejandra Rodríguez ${ }^{2}$ y Josep M. Campistoß ${ }^{3}$.

Servicio de N efrología ${ }^{1}$, Hospital Universitario Insular de Las Palmas de G ran Canaria. Servicio de N efrología, Hospital Universitario N uestra Señora de Candelaria², Tenerife y Servicio de N efrología y Trasplante Renaß, Hospital Clinico IDIBAPS, Barcelona. España

Resumen.- Las enfermedades glomerula res representan una causa importante y frecuente de pérdida de injertos renales a medio-largo plazo, especialmente por recidiva de la enfermedad renal primaria. En el trasplante renal de donante vivo, las enfermedades glomerulares tienen una connotación especial por el riesgo de recidiva de la enfermedad primaria con la consiguiente pérdida del injerto renal, y por el riesgo del donante de desarrollar una enfermedad glomerular postnefrectomía por su similitud con el receptor.

La incidencia de recidiva de la enfermedad glomerular postrasplante varía según cada tipo, siendo especialmente frecuente en la nefropatía lgA y en la glomerulonefritis memebrana proliferativa tipo II. Siempre se debe diferenciar entre la recidiva histológica y la clínica, siendo mucho más frecuente la primera. La práctica de

J. M. C a mpistol

Servicio de N efrología y Trasplante Renal Hospital C línico de Barcelona

C/ Villarroel, 170

08036 Barcelona. (España) una biopsia renal constituye la prueba diagnóstica esencial para detectar y confirmar la presencia de una enfermedad glomerular postrasplante, siendo necesario la práctica de un estudio mediante inmunofluorescencia para determinar la variedad de glomerulonefritis.

Palabras clave: G lomerulonefritis. Trasplante renal de donante vivo. Recidiva.

Summary.- G lomerular diseases are an important and frequent cause of renal transplant graft loss in the mid-long term, mainly due to primary renal disease recurrence. G lomerular diseases have particular connotations in living donor kidney transplantation, due to the risk of primary disease recurrence and subsequent graft loss, and also the risk of development of glomerular disease related donors have for their genetic similitude. The incidence of glomerular disease recurrence after transplantation varies with type, being especially frequent in $\lg A$ nephropathy and type II membranous proliferative glomerulopathy. The difference between histological and clinical recurrence should always be established, being much more frequent the first Renal biopsy is the essential diagnostic test to detect and confirm the existence of glomerular disea se after transplant, with immunofluo rescence study being necessary to determine the type of glomerular disease.

Keywords: Glomerular diseases. Living donor kidney transplantation. Recurrence. 


\section{INTRODUCCIÓN}

La pérdida del injerto renal por recidiva de la glomerulopatía (GM) de base se describió por primera vez en 1955, en un paciente con enfermedad renal terminal (ERT) secundaria a vasculitis que desarrolló una glomerulonefritis ( $G M N$ ) rápidamente progresiva después del trasplante renal. El desarrollo de GM postrasplante puede ser causado por:

a) Recidiva de la enfermedad de base.

b) Desarrollo de una nueva GM .

c) Trasplantar un injerto renal de un donante con una GM no diagnosticada.

Para afirmar que nos encontramos ante una recidiva de la GM hay tres condiciones que se deben cumplir sine qua non:

a) Conocer la enfermedad primaria del receptor.

b) GM del injerto.

c) Ausencia de enfermedad renal primaria en el donante (1).

La incidencia de recidiva de las GM postrasplante se estima entre un 6-19.4\% de los receptores de injerto renal, incrementándose la prevalencia con el tiempo de seguimiento. Para la mayoría de las GM, diagnosticar la recidiva de en el injerto renal puede ser difícil, siendo en general las GM de novo más frecuentes que las recidivas. 0 tra dificultad añadida es diferenciar entre GM y rechazo crónico, que con frecuencia cursa con alteraciones glomerulares. La presencia de alteraciones en el intersticio y espacio vascular, los hallazgos inmunohistológicos y la presentación clínica deberían permitir establecer el diagnóstico diferencial (1).

Se conoce la recidiva histológica postrasplante de todas las GMN, pero la incidencia y severidad de la recidiva clínica varía enormemente según el tipo de enfermedad glomerular (Tabla I). A mplios registros informan de que entre el $5-10 \%$ de los injertos fracasan por recidiva de la enfermedad primaria (2). En un estudio retrospectivo basado en los datos del Renal Allograft Disease Registry (RADR) se evaluó la prevalencia y el impacto de la recidiva y enfermedad de novo de las GM en el injerto. Se diagnosticaron 167 casos de GM de novo o recidivas mediante biopsia renal. Las formas anatomopatológicas por orden de frecuencia fueron: GMN focal y segmentaria (GMNFS), GMN IgA, nefropatía diabética, GMN

TABLA I. REC IDIVA DE G LO M ERULO N EFRITIS PRIM ARIA EN EL TRASPLAN TE. (KAHAN B, PON TICEU C. PRIN CIPES AN D PRAC TIC E O F REN AL TRAN SPLAN TIO N. SELEC TIO N AN D PREPARATIO OF THE REC IPIEN T.)

\begin{tabular}{|l|c|c|c|}
\hline ENFERMEDAD GLOMERULAR & Recidiva histológica (\%) & Recidiva clínica (\%) & Pérdida del injerto (\%) \\
\hline GM N focal & $20-50$ & $15-50$ & $10-40$ \\
\hline GM m membranosa & $5-10$ & $5-10$ & $1-5$ \\
\hline $\begin{array}{l}\text { GM N membrana-proliferativa } \\
\text { tipo I }\end{array}$ & $\begin{array}{c}15-30 \text { (adultos) } \\
20-60 \text { (niños) }\end{array}$ & $\begin{array}{c}20-25 \text { (adultos) } \\
10-60 \text { (niños) }\end{array}$ & $\begin{array}{c}10 \text { (adultos) } \\
10 \text { (niños) }\end{array}$ \\
\hline $\begin{array}{l}\text { GM N membrana-proliferativa } \\
\text { tipo II }\end{array}$ & $\begin{array}{c}85 \text { (adultos) } \\
95-100 \text { (niños) }\end{array}$ & $\begin{array}{c}15 \text { (adultos) } \\
25 \text { (niños) }\end{array}$ & $\begin{array}{c}10 \text { (adultos) } \\
20 \text { (niños) }\end{array}$ \\
\hline GM N Ig A & 100 (a largo plazo) & $30-50$ & $15-30$ (a los 5 años) \\
\hline $\begin{array}{l}\text { Enfermedad antimembrana } \\
\text { basal }\end{array}$ & $10-30$ & $<10$ & Raro \\
\hline
\end{tabular}


membranoproliferativa (MBP) y GMN membranosa $(\mathrm{MB})$, siendo la vida media del injerto más corta en las recidivas por GMN FS. Entre las condiciones demográficas asociadas con la recidiva de las GM destacan:
a) Sexo masculino.
b) Retrasplante.
c) Edad joven.

La aparición de GM de novo o por recidiva se asoció con una supervivencia más corta a largo plazo, multiplicándose por dos el riesgo relativo de pérdida del injerto (3).

\section{ASPECTOS ESPECÍFICOS DE LAS ENFERME- DADES GLOMERULARES Y DEL TRASPLANTE RENAL DE VIVO}

El trasplante de donante vivo merece especial atención por el riesgo de recidiva que puede conducir a pérdidas del injerto de forma precoz y, por el riesgo incrementado de algunas $\mathrm{GM}$ familiares, con consecuencias posteriores para ambos (donante y receptor) (1). No se han descrito diferencias significativas en la prevalencia de GM (de novo o por recidiva) entre el trasplante de donante vivo y de cadáver (3). Sin embargo, la pérdida del injerto por recidiva de la GM es más frecuente en el injerto de donante vivo que de donante cadáver (1). El riesgo de una nefrectomía unilateral no debería infraestimarse. La tasa de mortalidad perioperatoria se estima en alrededor de 1 de cada 3000 nefrectomías de donante vivo, sin embargo, no se ha descrito, con respecto a la población en general, mayor mortalidad a largo plazo $(4,5)$. La hipertensión e insuficiencia renal son potenciales riesgos asociados a la donación de un riñón (6).

La posibilidad de desarrollar enfermedad renal primaria en el donante vivo es un hecho que no hay que olvidar. Aquellos potenciales donantes con historia familiar de enfermedad renal primaria conocida que se transmita genéticamente, se debe de descartar con las máximas garantías la presencia de esta enfermedad en el donante. Uno de los síndromes en el que las anormalidades estructurales se han identificado pero en el que el polimorfismo genético y la heterogeneidad en la presentación clínica hace difícil predecir el riesgo renal es el síndrome de Alport. El consentimiento informado de un donante vivo incluye información sobre el riesgo social, quirúrgico y morbi- mortalidad a corto y largo plazo. De hecho, la determinación del posible riesgo para la salud incluye una evaluación médica enfocada en los factores de riesgo del donante, los relacionados con la nefrectomía, enfermedad médica y enfermedad renal primaria (4).

\section{GLOMERULOPATIAS ESPECÍFICAS}

\section{G lomerulonefritis segmentaria y focal (GMNFS)}

La GMNFS es una causa frecuente de ERT en adultos, apareciendo en $7-15 \%$ de los pacientes que requieren terapia renal sustitutiva. La tasa de recidiva se ha estimado entre el 20-30\%. Ante la aparición de proteinuria a las pocas horas o semanas postrasplante debemos sospechar la recidiva de la G M NFS. Como factores de riesgo, se han descrito: a) edad < 15 años; b) evidencia de proliferación mesangial en el riñón nativo; c) evolución hacia ERT en menos de 3 años; y d) pacientes con recidiva previa (estimándose la probabilidad entre 75-85\%). En pacientes sin factores de riesgo, la recidiva se estima entre un $10-15 \%$ (1). La recidiva de dicha $\mathrm{GMN}$ supone la pérdida del injerto en un $40-50 \%(6)$.

La GMNFS de novo es una lesión pobremente definida que frecuentemente se ve asociado con otros cambios, tal como nefroptía crónica del injerto o toxicidad por ciclosporina. El daño isquémico, la hiperfiltración, el rechazo crónico y la toxicidad por ciclosporina pueden jugar un papel importante en la patogénesis de dicha GM (7). Los pacientes que desarrollan recidiva de GMNFS presentan un riesgo incrementado de rechazo agudo, postulándose que juega un papel importante en dicha patogenia la presencia de un factor albuminúrico circulante, por lo que el uso de injertos de donante vivo debe ser discutido $(8,9)$. Un reciente estudio muestra que en pacientes pediátricos, el beneficio de un donante vivo se pierde si la enfermedad de base es una GMNFS (10). Los pacientes en edad pediátrica cuya enfermedad primaria es una GMNFS tienen, como media, un 90\% más de posibilidades de perder el injerto si se trata de un injerto renal de donante vivo y un $50 \%$ si se trata de un donante cadáver comparado con el receptor con otra enfermedad de base (10).

Se deberían evitar utilizar injertos de donante vivo si el curso clínico de la GMNFS es de menos de 3 
años de evolución o si el paciente tiene menos de 15 a ños de edad y anatomopatológicamente se evidencia proliferación mesangial difusa. Si el primer trasplante fracasa por recidiva de la enfermedad debería evitarse un segundo trasplante de vivo relacionado ya que la tasa de recidiva se estima en un $80 \%$. Por el contrario, si el primer injerto se perdió por rechazo 0 alguna otra complicación sin evidencia de recidiva, hay poco riesgo de recidiva en un segundo trasplante (10). Se han descrito tres casos de GMNFS y ERT en donantes que habían donando un riñón para un hermano con GMNFS (13). Por tanto, las recomendaciones según la guía de trasplante de donante vivo es que el trasplante de donante vivo debería evitarse en niños con GMNFS y adultos con GMNFS de rápida evolución, pero que el retrasplante de donante vivo es razonable si el primer injerto presentó una vida prolongada 0 estuvo libre de GMNFS (12). En caso de donante vivo, la posibilidad de recidiva precoz que conduzca a pérdida del injerto debería explicársele al potencial donante (14).

\section{G lomerulonefritis $\lg \mathrm{A}$}

La GMN IgA suele cursar con la presencia de microhematuria y proteinuria no nefrótica a los 2 meses - 4 años postrasplante, estimándose entre 25 $60 \%$ la prevalencia de recidiva. La pérdida del injerto por este motivo se ha descrito en raras ocasiones $(<10 \%)(1)$. La GMN IgA tiene un componente genético, que puede actuar como factor de riesgo para la recidiva en los injertos de donante vivo. El riesgo de recidiva está relacionado con el tiempo de seguimiento postrasplante, siendo casi del $100 \%$ a los $10-20$ años. La recidiva histológica de la GM IgA se observa en el $60 \%$ de los injertos renales en pacientes con nefropatía IgA primaria (12). Se sabe que los pacientes con nefropatía IgA tienen un mayor riesgo de recidiva de la enfermedad cuando reciben un riñón de un donante vivo relacionado (13), sin embargo, la tasa de recidiva en estos casos es tan baja que en la práctica pueden ser usados, aunque una evaluación familiar completa incluyendo una biopsia pretrasplante debería considerarse (14).

La Púrpura Schönlein Henoch y la GMN IgA probablemente representan la misma enfermedad en dos formas de expresión distintas. De los pocos datos que existen parece que los órganos de donante vivo no deberían utilizarse por el riesgo de recidiva que presentan, aunque un intervalo libre de enfermedad de al menos 8-12 meses reduce este riesgo (1).

\section{G lomerulonefritis membranoproliferativa}

La mayoría de los pacientes que presentan GMN Mesangiocapilar tipo I evolucionan lentamente hacia ERT y la recurrencia de esta enfermedad se estima entre un 20-30\% de los receptores del injerto. De éstos el $40 \%$ pierden el injerto renal. Dada la incidencia estimada de recidiva está en controversia el uso de injerto de donante vivo (1).

La GMN Mesangiocapilar tipo II recidiva, normalmente dentro del primer año, en el $50-100 \%$ de casos, siendo la probabilidad de pérdida del injerto del $10-20 \%$ (1). La mayoría de los pacientes con tipo II muestran recidiva histológica. La recidiva clínica es menos común, aproximadamente en el $10 \%$ de los adultos y en el $28 \%$ de los niños (12). Este riesgo se ve incrementado en un $50 \%$ en pacientes con los siguientes factores de riesgo: i) Varones; ii) Proteinuria en rango nefrótico; y iii) $\mathrm{GMN}$ rápidamente progresiva con proliferación extracapilar.

\section{Glomerulonefritis membranosa}

El conocer la verdadera incidencia de recidiva de la GMN membranosa es complicado por ser esta GMN la forma de aparición más común de GMN de novo en el injerto (2-9\%). Clínicamente la recidiva aparece de forma más precoz (1 semana a 2 años) con rápido desarrollo de síndrome nefrótico, mientras que la GMN de novo es más tardía (4 meses a 6 años). A pesar del bajo riesgo absoluto de recidiva, el 50-60\% de los injertos con recidiva presentan disfunción del mismo (1). La incidencia de recidiva es también elevada en los injertos de donante vivo $(11.9 \%$ vs $3 \%$ de injerto de cadáver) (15). La GMN membranosa de novo debería considerarse en casos de proteinuria y síndrome nefrótico después del trasplante, debiéndose excluir infecciones virales tales como VHC (16).

Se ha demostrado una asociación entre el alelo TAP1B y la GMN membranosa, quedando por determinar la posible relación de este marcador genético con la recidiva de dicha GMN. Se han descrito 
casos de recidiva precoz de esta $\mathrm{GMN}$ en receptores de injerto de donante vivo relacionados con HLA idéntico, por lo cual el uso de estos injertos debería considerarse cuidadosamente (1). El tiempo de recidiva también parece ser más precoz en receptores de injerto de donante vivo $(9.3+/-3$ vs. $18.2+/-7.5$ potraplante) (17).

\section{Enfermedad por anticuerpos antimembrana basal}

La tasa de recidiva histológica de la enfermedad por anticuerpos antimembrana basal en los trasplantados se define como la presencia de depósito de IgG en la membrana basal, apareciendo en alrededor del $50 \%$ si el trasplante se realiza en presencia de anticuerpos circulantes. Sólo un $25 \%$ de estos pacientes desarrollarán enfermedad clínica. La tasa de recidiva se estima en un 5-15\% si el trasplante se realiza 6 meses después de que los anticuerpos sean indetectables en suero (1). En GM por anticuerpos antimembrana basal, se recomienda esperar para la inclusión en lista de espera que los anticuerpos circulantes medidos por técnicas específicas hayan desaparecido por completo. La GM antimembrana basal tiende a recidivar sólo en pacientes con anticuerpos circulantes (11).

\section{CONCLUSIÓN}

En conclusión, los donantes vivos relacionados deberían evaluarse con particular atención pudiendo requerir una biopsia previa a la donación en casos de enfermedad glomerular familiar potencial. Los donantes vivos relacionados se aceptan de forma general en GMN MBP (tipo I y II), GMN MB, $N$ efropatía IgA y nefritis anti-MBG. Si un injerto previo se ha perdido por cualquier tipo de $G M N$ recidivante en el pasado, el injerto de donante vivo debería reservarse para circunstancias excepciona les ya que el riesgo para una segunda recidiva y pérdida del injerto parece muy alta (18).

\section{BIBUOGRAFIA y LECTURAS RECOMENDADAS (*lectura de interés y **lectura fundamental)}

**1. KOTANKO, P.; PUSEY, C.D.; LEVY, J.: "Recurrent glomerulonephritis following renal transplantation". Transplantation, 63: 1045, 1997.
**2. DENTON, M.D.; SINGH, A.K.: "Recurrent and de novo glomerulonephritis in the renal allograft". Semin. Nephrol., 20: 164, 2000.

**3. HARIHARAN, S.; ADAMS, M.B.; BRENNAN, D.: "Recurrent and de novo glomerular disease after renal transplantation: A report from Renal Allograft Disease Registry (RADR)". Transplantation, 68: 635, 1999.

*4. DAVIS CONNIE, L.: "Evaluation of the living kidney donor: Current perspectives". American Journal Kidney Disease, 43: 508, 2004.

*5. HARTMANN, A.; FAUCHAD, P.; WESTLIE, L.: "The risk of living kidney donation". Nephrol Dial Transplant, 18: 871, 2003.

*6. ARTERO, M.; BIAVA, C.; AMEND, W.: "Recurrent focal glomerulosclerosis: natural history and response to therapy". Am. J. Med., 92: 375, 1992.

*7. STEPHANIAN, E.; MATAS, A.J.; MAUER, S.M.: "Recurrence of disease in patients retransplanted for focal segmental glomerulosclerosis". Transplantation, 53: 755, 1992.

*8. YAKUPOGLU, U.; BARANOWSKA DACA, E.; SUKI, W.N.: "New aspects of posttrasplant nephrotic syndrome: clinicopathologic correlations with outcomes". Transplantation Proc., 36: 139, 2004.

*9. BAUM, M.A.; STABLEIN, D.M.; PANZARINO, V.M. y cols.: "Loss of living donor renal allograft survival advantage in children with focal segmental glomerulosclerosis". Kidney Int., 59: 328, 2001.

**10. NEWSTEAD, C.: "Recurrent disease in renal transplants". Nephrology Dialysis Transplantation, 18: 68, 2003.

*11. ISMALL ALLONCH, M.; BURKE, G.; NERY, J.: "Rapidilly progressive focal segmental glomeruloesclerosis occurring in a living related kidney transplant donor. Case report and review of 21 cases of kidney transplant for primary FSGS". Transplant Proc., 125: 2176, 1993.

*12. EBGP (European Expert Group on Renal Transplantation); European Renal Association (ERAEDTA); European Society for Organ Transplantation (ESOT): "Evaluation, selection and preparation of the potential transplant recipient. Recurrence of original renal disease". Nephrol Dial Transplant, 15: 11, 2000.

*13. ANDRESDOTTIR, M.; ASSMANN, K.; HOITSMA, A.: "Recurrence of type I membranoproliferative glomerulonephritis after renal transplantation: analysis of the incidence, risk factors, and impact on graft survival" Transplantation, 63: 1628, 1997.

*14. BRENSILVER, J.M.; MALLAT, S.; SCHOLES, J.: "Recurrent $\operatorname{IgA}$ nephropaty in living related donor transplantatio: recurrence or transmission of familial disease?”. Am. J. Kidney Dis., 12: 147, 1998.

*15. DAVISON, A.M.; JOHNSTON, P.A.: "Allograft membranous nephropaty”. Nephrol Dial Transplant, 7: 114, 1992.

*16. European best practice guidelines for renal transplantation. Section IV: Long-term management of the transplant recipient. IV. 2.4. Chronic graft dysfunction. De novo renal disease after transplantation.

*17. JOSEPHSON, M.A.; SPARGO, B.: "The recurrence of membranous glomerulopathy in a renal transplant recipient”. Am. J. Kidney Dis., 24: 873, 1994.

**18. FLOEGE JÜRGEN.: "Recurrent glomerulonephritis following renal transplantation:an update". Nephrol Dial Transplant, 18: 1260, 2003. 